\title{
Vivência acadêmica e desempenho acadêmico de ingressantes em cursos de computação
}

\author{
Evoney Mendonça ${ }^{1}$, Leandro S. G. Carvalho ${ }^{1}$, Ana Lucia Machado dos Santos ${ }^{1}$, \\ Elaine H. T. Oliveira ${ }^{1}$, David B. F. Oliveira ${ }^{1}$, Filipe Dwan Pereira ${ }^{2}$ \\ Instituto de Computação - Universidade Federal Amazonas (UFAM) \\ ${ }^{2}$ Departamento de Ciência da Computação - Universidade Federal de Roraima (UFRR) \\ evoney.mendoncadicomp.ufam.edu.br
}

\begin{abstract}
Resumo. Este estudo investiga como as vivências acadêmicas de estudantes de cursos de computação podem influenciar no desempenho dos alunos. A vivência acadêmica foi mensurada em 5 dimensões (Carreira, Pessoal, Institucional, Interpessoal, Estudo) utilizando o Questionário de Vivências Acadêmicas em sua versão reduzida (QVA-r) como um instrumento de previsão do desempenho de estudantes. $O$ QVA-r foi aplicado por dois anos a ingressantes em cursos de graduação em computação de uma universidade pública $(N=266$ alunos $)$. Os resultados não sugeriram correlação significativa entre desempenho e fatores, do QVA-r, porém os fatores explicam $89,9 \%$ da variação do desempenho do aluno (valor- $p<0,05$ ).
\end{abstract}

\begin{abstract}
This article investigates how the academic experiences of students in computing courses can influence student performance. The academic experience was measured in 5 dimensions (Career, Personal, Institutional, Interpersonal, Study) using the Academic Experience Questionnaire in its reduced version (AEQ-r) as an instrument for predicting student performance. The AEQ-r was applied for two years to newcomers to undergraduate courses in computing at a public university ( $N=266$ students). The results did not suggest a signifcant correlation between performance and factors of the AEQ-r, but the factors explained $89.9 \%$ of the variation in student performance $(p-$ value $<0.05)$.
\end{abstract}

\section{Introdução}

Nos estudos de evasão no ensino superior, observa-se que o primeiro ano tem impacto sobre a permanência do estudante no curso de graduação [Brito et al. 2015, Digiampietri et al. 2016, Zanato et al. 2018, Damasceno and Carneiro 2018]. Por exemplo, [Oliveira Júnior et al. 2017] encontraram que $80 \%$ das desistências nos cursos de graduação presencial de sua instituição acontecem até o $3^{\circ}$ período, independente da duração total do curso.

E por que o primeiro ano é tão impactante? Conforme [Oliveira et al. 2014], no processo de adaptação acadêmica, o estudante universitário vivencia novas exigências e responsabilidades do ensino superior, tais como: postura ativa frente à aprendizagem, ajustamento às regras da instituição, bem como convívio com novos colegas e professores.

Do ponto de vista do estudante, podemos entender a adaptação acadêmica pela forma como ele(a) mobiliza atitudes para enfrentar as novas pressões externas: gerenciamento do tempo, capacidade de estabelecer novas amizades, grau de estresse com que 
reage às demandas acadêmicas, vínculo com a instituição, descoberta da sua vocação, entre outras [Oliveira et al. 2014, Granado et al. 2005]. Ou seja, a adaptação acadêmica não está unicamente vinculada a competências técnicas e instrumentais, mas também a competências de ordem afetiva e social.

Ainda que passado o primeiro ano de curso, os estudantes continuam sendo afetados por outras pressões durante sua permanência no curso. A esse conjunto de situações ou variáveis próprias do contexto de vida dos estudantes universitários, do qual dependem o desenvolvimento pessoal, cognitivo e social do indivíduo, [Almeida et al. 2002] e [Granado et al. 2005] se referem por vivências acadêmicas.

Para estimar como essas experiências interferem na vida acadêmica de estudantes de três cursos de Computação da Universidade < omitido para revisão $>$, foi aplicado o Questionário de Vivências Acadêmicas na versão reduzida (QVA-r), adaptada ao português brasileiro por [Granado et al. 2005]. O QVA-r é um instrumento de autorrelato que avalia as percepções dos estudantes em relação às suas experiências acadêmicas no curso superior. É composto por 55 itens, em escala Likert com cinco níveis de resposta, agrupados em cinco dimensões: Pessoal, Interpessoal, Carreira, Estudo e Institucional (mais detalhes são apresentados na Seção 3.1).

O objetivo deste artigo é verificar se existe relação entre as dimensões do QVAr e o desempenho dos estudantes nos dois primeiros períodos letivos, apontados como decisivos na literatura para a permanência do estudante em seu curso. Em específico, este trabalho visa responder as seguintes questões de pesquisa:

QP1: Existe correlação significativa entre cada dimensão do QVA-r e o desempenho acadêmico do estudante?

QP2: Até que ponto um modelo de regressão pode explicar a variação do desempenho a partir das dimensões do QVA-r?

\section{Trabalhos relacionados}

No contexto do ensino superior presencial, o QVA-r é um instrumento psicométrico validado que tem sido amplamente utilizado em diversas pesquisas relacionadas à adaptação universitária e permanência no curso. Dentre elas, foi verificada correlação significativa do QVA-r com outras escalas, tais como: desenvolvimento de carreira [Mognon and Santos 2013]; empregabilidade [Lamas et al. 2014]; interesses profissionais [Noronha et al. 2009]; adaptabilidade de carreira e de motivos para evasão do ensino superior [Ambiel et al. 2016]; questionário de dados sociodemográficos [Sarriera et al. 2012]; e questionários sociodemográfico e de envolvimento acadêmico [Porto and Soares 2017].

Apesar dessa ampla produção científica em torno do QVA-r, nota-se uma ausência de estudos que tentam associar as dimensões do QVA-r com o desempenho acadêmico do estudante. Além disso, observa-se a importância de tentar analisar como as dimensões do QVA-r podem influenciar no desempenho dos alunos. Em um exemplo de estudo que busca essa relação questionário-desempenho, [Rodrigues et al. 2017] utilizaram outro instrumento, o Online Self-regulated Learning Questionnaire (OSLQ), para estimar o desempenho de estudantes de quatro cursos de graduação na modalidade de educação a distância (EaD), por meio de um modelo de regressão logística. 
O OSLQ avalia habilidades de autorregulação, característica importante na EaD, a qual exige do aluno uma maior autonomia no processo de aprendizagem. Dessa forma, o presente estudo se alinha com os objetivos de [Rodrigues et al. 2017], mas utiliza as dimensões do QVA-r como variáveis independentes do modelo de regressão, pois é um instrumento mais adaptado ao ensino presencial e já validado nos diversos trabalhos já citados.

Quanto aos cursos estudados, o QVA-r já foi aplicado a estudantes de engenharia [Santos et al. 2006], enfermagem [Araújo et al. 2011], psicologia [Igue et al. 2008], medicina [Anjos and Aguilar-da Silva 2017], administração e direito [Noronha et al. 2009], entre outros. No melhor do nosso conhecimento, o presente estudo é o primeiro que analisa a aplicação do QVA-r a estudantes de Computação.

\section{Metodologia}

\subsection{Instrumento}

O Questionário de Vivências Acadêmicas, versão reduzida (QVA-r), foi criado por [Almeida et al. 2002] e adaptado para o português brasileiro por [Granado et al. 2005]. É um instrumento de autorrelato, composto por 55 itens, com resposta em escala Likert de 5 pontos. Foi escolhido por requerer um menor tempo de aplicação (cerca de $15 \mathrm{~min}$ ) em relação ao QVA (170 itens, média de $30 \mathrm{~min}$ ). Os itens estão distribuídos em 05 dimensões [Granado et al. 2005]:

1. Pessoal (14 itens): avalia o bem estar físico e psicológico dos alunos, o seu equilíbrio emocional, a estabilidade afetiva, o otimismo e a sua autoconfiança;

2. Interpessoal (12 itens): avalia as relações com os colegas, as competências de relacionamento em situações de maior intimidade, o estabelecimento de amizades e a procura de ajuda;

3. Carreira (12 itens): avalia os sentimentos relacionados com o curso, as perspectivas de carreira e os projetos vocacionais dos alunos;

4. Estudo (09 itens): avalia os hábitos de estudo, a gestão do tempo, a utilização dos recursos de aprendizagem no campus e a preparação para os testes; e

5. Institucional (08 itens): integra a apreciação dos alunos face à instituição de ensino que frequentam, o desejo de permanecer ou mudar de instituição, o conhecimento e a apreciação das infraestruturas existentes.

\subsection{Procedimentos}

O QVA-r foi aplicado aos alunos ingressantes em três cursos de graduação presencial na área de Computação da Universidade < omitido para revisão $>$, no início do segundo semestre letivo de 2018 e 2019. Ou seja, em cada ano, a aplicação se deu após um semestre letivo de vivência dos estudantes no ambiente acadêmico.

Em cada curso, apurou-se a turma com maior número de calouros matriculados. Depois, pediu-se aos professores responsáveis que cedessem um tempo de aula para aplicação do instrumento. Os estudantes foram sensibilizados sobre a importância da pesquisa e convidados a preencher o QVA-r, cadastrado na plataforma Moodle da Instituição.

Os calouros ausentes ou não matriculados nas turmas foram contactados posteriormente por e-mail e convidados a preencher o questionário no momento mais conveniente, 
mas dentro de um prazo informado. Antes de acessar a tela do QVA-r, um Termo de Consentimento Livre e Esclarecido (TCLE) foi apresentado aos estudantes. O estudo foi submetido ao Comitê de Ética em Pesquisa com seres humanos, mas foi considerado sem objeto por apresentar caráter censitário.

Para determinar a pontuação de cada dimensão do QVA-r, primeiramente foram recodificados os itens elaborados em escala Likert reversa. Em seguida, calculou-se a média aritmética das respostas aos respectivos conjuntos de itens.

Para responder às questões de pesquisa, os cinco fatores do QVA-r foram utilizados como variáveis independentes e o desempenho acadêmico como variável de resposta. Para representar o desempenho, foram escolhidas duas métricas: os coeficientes de rendimento do $1^{\circ}$ e $2^{\circ}$ período letivo (CR-1P e CR-2P). O CR é entendido como a média aritmética das disciplinas cursadas em determinado período. Não é acumulativo.

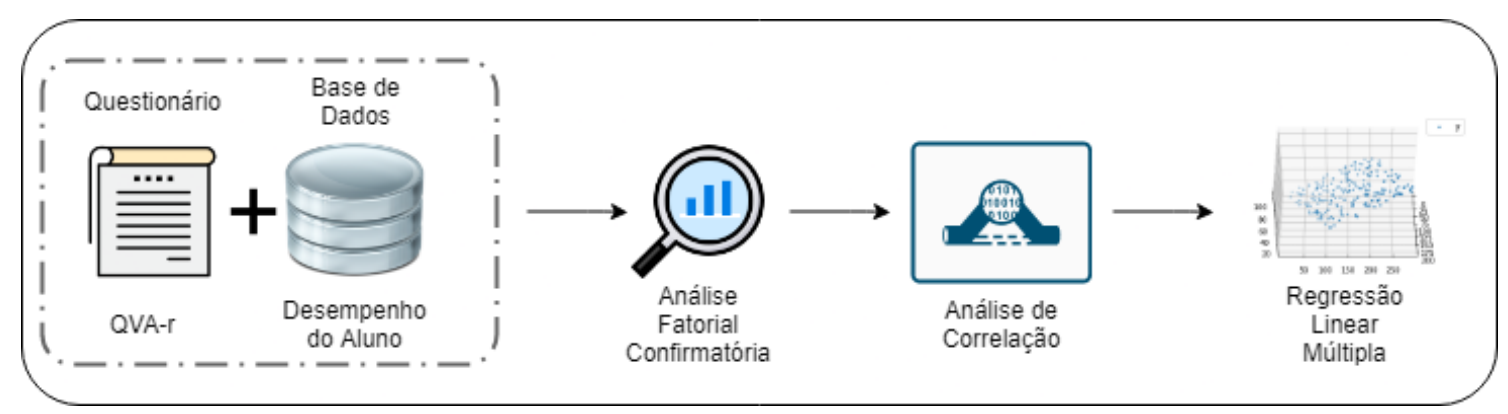

Figura 1. Procedimentos de análise dos dados.

Como mostra a Figura 1, após a coleta dos dados acadêmicos, realizou-se a análise fatorial confirmatória das respostas ao QVA-r. Em seguida, após o fim do segundo semestre de cada ano letivo, foram verificadas as correlações entre as dimensões do QVA-r e as métricas de desempenho (CR-1P, CR-2P). Por fim, foram testados 5 modelos de regressão linear múltipla, cada um tendo como variável de resposta uma das 5 métricas de desempenho consideradas, conforme esta equação estrutural:

$$
Y_{i}=\alpha_{i 0}+\alpha_{i 1} D_{1}+\alpha_{i 2} D_{2}+\alpha_{i 3} D_{3}+\alpha_{i 4} D_{4}+\alpha_{i 5} D_{5}+\epsilon_{i}
$$

onde $Y_{i}, i=\{1,2\}$, representa as duas métricas de desempenho (CR-1P e CR-2P); $D_{1} \ldots D_{5}$ representam as dimensões do QVA-r (Pessoal, Interpessoal, Carreira, Estudo e Institucional); $\alpha_{i 1} \ldots \alpha_{i 5}$ representam os coeficientes de regressão; a constante $\alpha_{i 0}$ representa o valor da nota quando todas as variáveis explanatórias têm valor nulo; e $\epsilon_{i}$ é o erro aleatório de cada modelo.

\section{Resultados}

\subsection{Caracterização da amostra}

A amostra era constituída por alunos de Engenharia da Computação, Ciência da Computação e Engenharia de Software, ingressantes em 2018 e 2019. Dos 163 ingressantes em 2018, 128 responderam o questionário, dos quais 105 (82,0\%) eram homens e $23(18,0 \%)$ mulheres. A média das idades era 20,0 anos, com desvio padrão de 4,2. Por outro lado, para amostra coletada do ano de 2019, com 179 ingressantes, participaram 
138 estudantes, com idade média de 20,1 anos e desvio padrão de 4,4, sendo $109(79,0 \%)$ homens e $29(21,0 \%)$ mulheres.

A Figura 2 apresenta a distribuição de frequência das principais características demográficas dos estudantes ingressantes em 2018 e 2019: sexo (masculino e feminino), idade, processo seletivo (PSC - vestibular da instituição, SISU - vestibular nacional, e outras formas de ingresso) e cota (AC - ampla concorrência, Renda - estudantes provenientes de escola pública com renda até 1,5 salários mínimos, Não Renda - estudantes provenientes de escola pública independentes de renda).
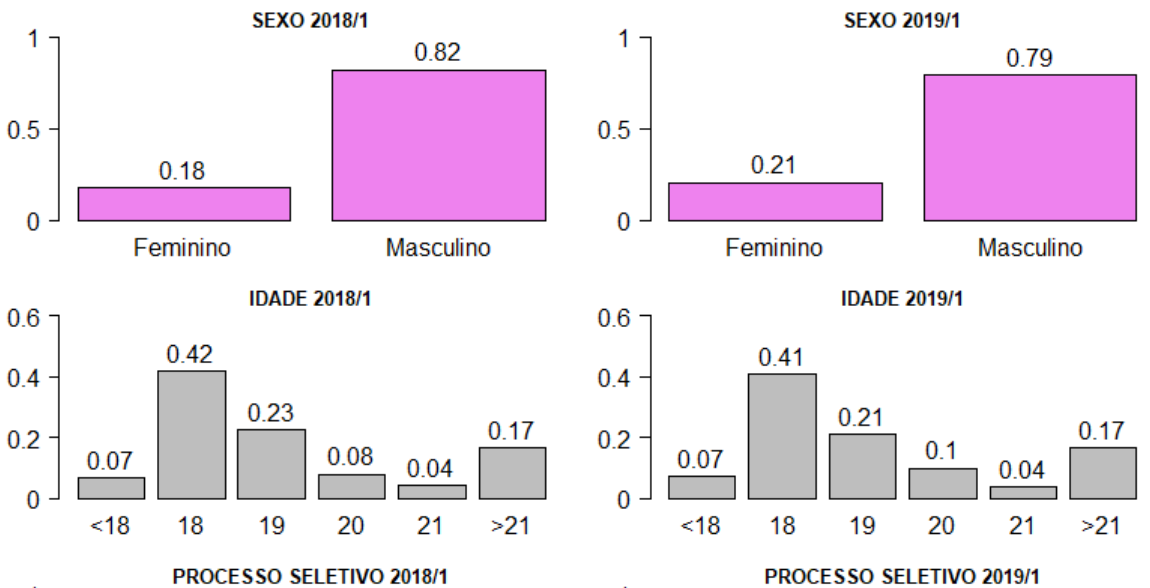

$p \approx 0.9579$
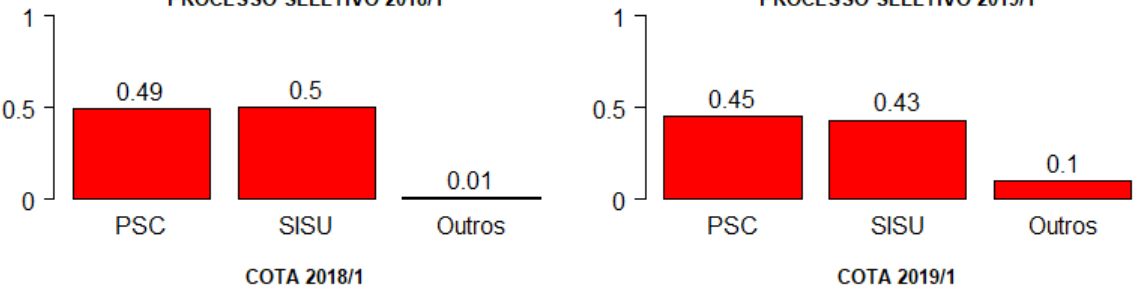

$p \approx 0.9557$
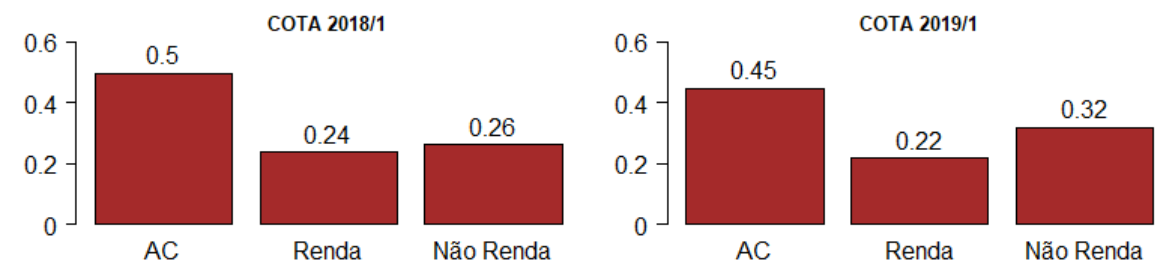

$p \approx 0.9905$

$\mathrm{p} \approx 0.9957$

Figura 2. Teste Qui-Quadrado entre as características demográficas dos estudantes respondentes

Para cada par de gráficos contidos na figura, à direita, é indicado o valor- $p$ do teste qui-quadrado que avaliou a semelhança das distribuições de frequência da característica evidenciada. Em todos os casos, não foi possível rejeitar a hipótese nula de que não há diferença significativa entre as amostras. Ou seja, as características demográficas dos estudantes que ingressaram em 2018 são semelhantes aos de 2019, o que nos permite considerar os dados como provenientes de uma amostra única.

\subsection{Análise fatorial confirmatória}

Para validar os resultados do QVA-r, seguiu-se a mesma metodologia adotada por [Marinho-Araujo et al. 2015]. Assim, foram empregados os seguintes procedimentos estatísticos: (1) análise de pressupostos para a análise fatorial confirmatória, considerando 
um nível de confiança de 95\%, por meio do teste de Kaiser-Mayer-Olkin (KMO), (2) teste de esfericidade de Bartlett, e (3) coeficiente alfa de Cronbach.

O KMO analisa as correlações parciais das variáveis, enquanto que o teste de esfericidade de Bartlett atesta a hipótese de que a matriz de covariâncias é uma matriz identidade [Tabachnick et al. 2007]. Por sua vez, o coeficiente alfa de Cronbach verifica a consistência interna dos itens do questionário, que no presente estudo foi realizada para a escala total. Os resultados são descritos na Tabela 1.

Tabela 1. Resultados dos pressupostos para análise fatorial confirmatória.

\begin{tabular}{lrr}
\hline \multirow{2}{*}{ Pressuposto } & \multicolumn{3}{c}{ Ano da amostra } \\
\cline { 2 - 3 } & \multicolumn{2}{c}{$\mathbf{2 0 1 8}$} \\
\hline Esfericidade de Bartlett & $p \leq 0,001, \chi^{2}=4111,977$ & $p \leq 0,001, \chi^{2}=4078,725$ \\
KMO (Kaiser-Meyer-Olkin) & 0,77 & 0,76 \\
Alfa de Cronbach & 0,92 & 0,92 \\
\hline
\end{tabular}

Dessa forma, tais resultados confirmam a adequação dos dados à análise fatorial confirmatória. Para tanto, foi feita a análise fatorial dos eixos principais com rotação oblíqua (oblimin), por se considerar as dimensões correlacionáveis [Marinho-Araujo et al. 2015]. Confirmou-se uma solução de cinco fatores com índices SRMR (média da discrepância entre a matriz amostral e a matriz estimada) inferiores a 0,08 (valor de corte sugerido por [Hu and Bentler 1999]) para as amostras de 2018 e 2019.

\subsection{Análise de correlação}

Para responder à QP1 (Existe correlação significativa entre cada dimensão do QVA-r e o desempenho acadêmico do estudante?), foram tomados dois conjuntos de medidas: as duas métricas de desempenho (CR-1P e CR-2P) e as médias de pontuação das respostas de cada dimensão do questionário (Pessoal, Interpessoal, Carreira, Estudo e Institucional). Em seguida, cada métrica de desempenho foi relacionada, por meio da correlação de Spearman ${ }^{1}$, com cada um dos fatores do QVA-r. Essa análise foi realizada sobre a junção das amostras coletadas nos anos de 2018 e 2019, uma vez que suas características demográficas são semelhantes (Figura 2).

De acordo com o gráfico da Figura 3, as dimensões do QVA-r que obtiveram maiores coeficientes de correlação de Spearman com desempenho acadêmico foram Estudo, Pessoal e Interpessoal, com valores- $p$ menores que 0,05, o que indica significância estatística para essas correlações. Porém, os coeficientes de correlação encontrados foram baixos $(<0,40)$, indicando uma correlação fraca.

Essa baixa correlação pode ser explicada pela multidimensionalidade inerente à adaptação do estudante à vida acadêmica, envolvendo fatores intrínsecos e extrínsecos à sua pessoa. Dessa forma, a próxima seção mostra uma análise utilizando a técnica de regressão linear múltipla, que objetiva a construção de um modelo multivariado, para responder à QP2 (Até que ponto um modelo de regressão pode explicar a variação do desempenho a partir das dimensões do QVA-r?).

\footnotetext{
${ }^{1}$ A correlação de Spearman avalia a relação monotônica entre duas variáveis ordinais, portanto, não exige que os dados provenham de uma distribuição normal.
} 


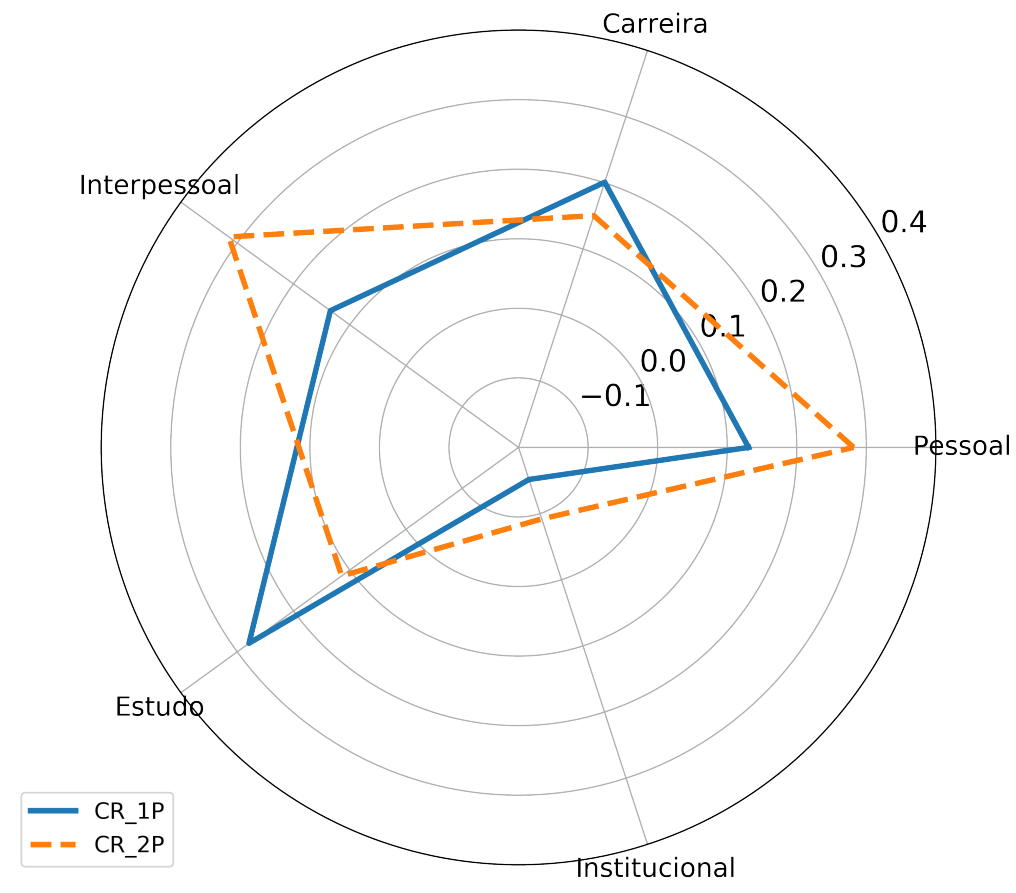

Figura 3. Análise de correlação entre as dimensões do QVA-r e as métricas de desempenho acadêmico

\subsection{Análise da regressão linear múltipla}

A regressão linear múltipla é uma técnica estatística usada para analisar a relação entre uma variável dependente e várias variáveis independentes explicativas. Essa técnica foi escolhida pelo fato de ser facilmente interpretável. Para construção do modelo, foi utilizada uma regressão com regularização, elastic net, e o método backward elimination para identificar quais dimensões do QVA-r obtiveram contribuição significativa ao modelo final, ou seja, aquelas dimensões com valores $p<0,05$.

A partir da Figura 4, verifica-se que as dimensões Carreira, Interpessoal e Estudo contribuem positivamente para o modelo envolvendo a variável dependente CR-1P, ou seja, quanto maior a pontuação da resposta do estudante para essas dimensões, melhor é o desempenho. A dimensão Carreira obteve os maiores coeficientes, indicando que alunos com visão mais clara sobre construção de carreira durante a academia tendem a ter melhores resultados em termos de desempenho. Entretanto, a dimensão Institucional contribuiu negativamente para o modelo, isto é, quanto maior a pontuação do estudante para essa dimensão, menor o seu desempenho acadêmico.

Para a variável dependente CR-2P, o modelo obteve a constante da equação estrutural (intercepto $\alpha_{0}$ ) como contribuidora significativa ao desempenho do estudante. Em um modelo de regressão, o intercepto representa o valor da variável dependente quando todas as variáveis independentes têm um valor nulo, ou seja, ela representa uma correção do viés de estimação dos coeficientes.

Por fim, nota-se que o modelo de regressão foi capaz de explicar 89,9\% da variação de CR-1P, mas apenas 17,9\% da variação de CR-2P. Como o QVA-r foi aplicado no início do segundo semestre letivo, provavelmente foi capaz de explicar a nota mais próxima no tempo, o $\mathbf{C R - 1 P}$, determinado no final do primeiro período letivo, do 
que prever a nota mais distante, o CR-2P, determinado quatro meses depois, no final do segundo período letivo.

Dessa forma, os resultados sugerem que um melhor momento de aplicação do QVA-r talvez seja antes do fim ou do primeiro período letivo, ou do segundo, a fim de que possa ser usado como instrumento de predição. Novos estudos devem ser conduzidos para verificar essas possibilidades.

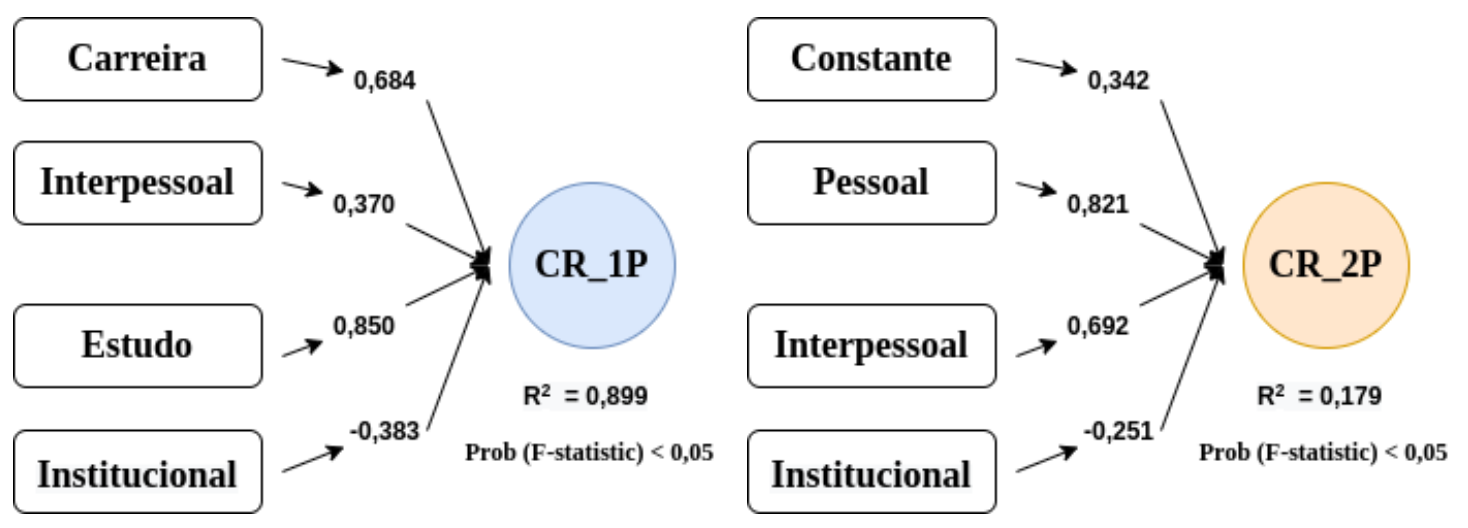

Figura 4. Coeficientes de regressão significativos (valor-p $<0,05$ ) nos modelos de regressão das métricas de desempenho em função das dimensões do QVA-r

\subsection{Limitações}

Este trabalho utilizou uma amostra por conveniência, ou seja, foram selecionadas amostras acessíveis da população, tanto pela disponibilidade de estudantes para responder o questionário, quanto pelo tempo da pesquisa. Talvez as informações dos alunos não respondentes pudessem enriquecer a análise. Além disso, por decorrência da pandemia da COVID-19, a realização dos semestres acadêmicos foi atrasada, impedindo a coleta de dados no mesmo momento em que foi feita em 2018 e 2019.

\section{Conclusão e trabalhos futuros}

Este artigo mostrou o potencial do QVA-r como instrumento que pode ser usado para explicar o desempenho acadêmico dos estudantes de computação, a partir da avaliação das suas vivências acadêmicas. Foram encontradas correlações fracas entre as dimensões do QVA-r e as métricas de desempenho (CR-1P e CR-2P) dos estudantes, porém foram correlações estatisticamente significantes. Também foi construído um modelo de regressão múltipla que explicou $89,9 \%$ de rendimento do primeiro período letivo (CR1P). Com isso, observa-se a importância de um bom acolhimento aos ingressantes de graduação com explicações sobre boa construção de carreira, educação emocional, bons hábitos de estudo, gestão de tempo, entre outros.

Como trabalho futuro, pretende-se refazer a análise de regressão com estratificação por características socio-demográficas, tais como cota, sexo, tipo de escola do ensino médio (pública ou privada), renda familiar, conhecimento prévio em programação, entre outras. O objetivo é identificar possíveis grupos com desempenho vulnerável por meio das dimensões do QVA-r. Outra abordagem interessante seria uma análise qualitativa das respostas a um item discursivo que foi adicionado ao final do QVA-r 
aplicado, usando processamento de linguagem natural, para depois verificar a relação dessas análises com as dimensões do QVA-r ou com as métricas de desempenho dos alunos.

\section{Agradecimentos}

Esta pesquisa, realizada no âmbito do Projeto Samsung-UFAM de Ensino e Pesquisa (SUPER), nos termos do artigo 48 do Decreto ${ }^{\circ}$ 6.008/2006 (SUFRAMA), foi parcialmente financiada pela Samsung Eletrônica da Amazônia Ltda., nos termos da Lei Federal $\mathrm{n}^{\mathrm{o}}$ 8.387/1991, por meio dos convênios 001/2020 e 003/2019, firmados com a Universidade Federal do Amazonas e a FAEPI, Brasil. O presente trabalho foi realizado com apoio da Coordenação de Aperfeiçoamento de Pessoal de Nível Superior - Brasil (CAPES) Código de Financiamento 001.

\section{Referências}

Almeida, L. S., Soares, A. P., and Ferreira, J. A. G. (2002). Questionário de Vivências Acadêmicas (QVA-r): Avaliação do ajustamento dos estudantes universitários. Avaliação Psicológica, 1(2):81-94.

Ambiel, R., Santos, A. A. A., and Dalbosco, S. (2016). Motivos para evasão, vivências acadêmicas e adaptabilidade de carreira em universitários. Psico, 47(4):288-297.

Anjos, D. R. L. and Aguilar-da Silva, R. H. (2017). Questionário de vivências acadêmicas (qva-r): avaliação de estudantes de medicina em um curso com currículo inovador. Avaliação: Revista da Avaliação da Educação Superior, 22(1):105-123.

Araújo, B., Vieira, M., Fernandes, A., and Sá, L. (2011). Determinantes psicossociais no percurso académico: estudo com estudantes de enfermagem.

Brito, D. M., Lemos, M., Pascoal, T. A., Rêgo, T. G., and Araújo, J. (2015). Identificação de estudantes do primeiro semestre com risco de evasão através de técnicas de data mining. Nuevas Ideas en Informática Educativa TISE, pages 459-463.

Damasceno, I. and Carneiro, M. (2018). Panorama da evasão no curso de sistemas de informação da Universidade Federal de Uberlândia: um estudo preliminar. In Simpósio Brasileiro de Informática na Educação (SBIE), volume 29, pages 1766-1770.

Digiampietri, L. A., Nakano, F., and Souza Lauretto, M. (2016). Mineraçao de dados para identificaçao de alunos com alto risco de evasão: Um estudo de caso. Revista de Graduação USP, 1(1):17-23.

Granado, J. I. F., Santos, A. A. A., Almeida, L. S., Soares, A. P., and Guisande, M. A. (2005). Integração académica de estudantes universitários: Contributos para a adaptação e validação do QVA-r no Brasil. Psicologia e Educação, 4(2):31-41.

Hu, L.-t. and Bentler, P. M. (1999). Cutoff criteria for fit indexes in covariance structure analysis: Conventional criteria versus new alternatives. Structural equation modeling: a multidisciplinary journal, 6(1):1-55.

Igue, É. A., Bariani, I. C. D., and Milanesi, P. V. B. (2008). Vivência acadêmica e expectativas de universitários ingressantes e concluintes. Psico-USF, 13(2):155-164.

Lamas, K. C. A., Ambiel, R. A. M., and Silva, B. T. A. O. L. (2014). Vivências acadêmicas e empregabilidade de universitários em final de curso. Temas em Psicologia, 22(2):329-340. 
Marinho-Araujo, C. M., Souza Fleith, D., Almeida, L. S., Bisinoto, C., and Rabelo, M. L. (2015). Adaptação da escala expectativas acadêmicas de estudantes ingressantes na educação superior. Avaliação Psicológica: Interamerican Journal of Psychological Assessment, 14(1):133-141.

Mognon, J. F. and Santos, A. A. A. (2013). Relação entre vivência acadêmica e os indicadores de desenvolvimento de carreira em universitários. Revista brasileira de orientação profissional, 14(2):227-237.

Noronha, A. P. P., Martins, D. F., Gurgel, M. G. A., and Ambiel, R. (2009). Estudo correlacional entre interesses profissionais e vivências acadêmicas no ensino superior. Psicologia Escolar e Educacional, 13(1):143-154.

Oliveira, C. T., Carlotto, R. C., Vasconcelos, S. J. L., and Dias, A. C. G. (2014). Adaptação acadêmica e coping em estudantes universitários brasileiros: uma revisão de literatura. Revista Brasileira de Orientação Profissional, 15(2):177-186.

Oliveira Júnior, J. G., Noronha, R. V., and Kaestner, C. A. A. (2017). Método de seleção de atributos aplicados na previsão da evasão de cursos de graduação. Revista de Informática Aplicada, 13(2).

Porto, A. M. S. and Soares, A. B. (2017). Diferenças entre expectativas e adaptação acadêmica de universitários de diversas áreas do conhecimento. Análise Psicológica, 35(1):13-24.

Rodrigues, R., Ramos, J. L. C., Silva, J., Araujo, T., Souza, H., Souza, F. F., Gomes, A. S., and Gouveia Zambom, E. (2017). Previsão de desempenho de alunos baseados em construtos de autorregulação da aprendizagem. In Simpósio Brasileiro de Informática na Educação (SBIE), volume 28, pages 1207-1216.

Santos, A. A. A., Suehiro, A. C. B., Oliveira, E. Z., Cunha, S. M., and Carrilho, D. M. (2006). Avaliação da integração acadêmica no ensino superior: estudo com estudantes de engenharia. Psicologia e Educação, 13(2):79-89.

Sarriera, J. C., Paradiso, Â. C., Schütz, F. F., and Howes, G. P. (2012). Estudo comparativo da integração ao contexto universitário entre estudantes de diferentes instituições. Revista Brasileira de Orientação Profissional, 13(2):163-172.

Tabachnick, B. G., Fidell, L. S., and Ullman, J. B. (2007). Using multivariate statistics, volume 5. Pearson Boston, MA.

Zanato, K. Y. S., Ventura, T. M., and Ribeiro, J. M. (2018). Análise da evasão de alunos da área de tecnologia da informação por meio de um banco de dados orientado a grafos. Rev. Eletr. Argentina-Brasil de Tecnologias da Informação e da Comunicação, 1(8). 